phys. stat. sol. (b) 205, 347 (1998)

Subject classification: $78.55 . \mathrm{Kz} ; 71.35 . \mathrm{Cc}$; S12

\title{
Electric Field Induced Luminescence Quenching in Conjugated Polymers and in Molecularly Doped Polymers
}

\author{
M. C. J. M. Vissenberg $(a, b)^{1}$ ) and M. J. M. DE Jong (a) \\ (a) Philips Research Laboratories, NL-5656 AA Eindhoven, The Netherlands \\ (b) Instituut-Lorentz, University of Leiden, NL-2300 RA Leiden, The Netherlands
}

(Received August 19, 1997)

\begin{abstract}
In conjugated polymer blends as well as in molecularly doped polymer systems the photoluminescence intensity decreases under application of an electric field. This effect is described using a theory that combines the migration of excitons with the dissociation of excitons into free charge carriers. Experimentally, clear differences between both systems have been observed when varying the concentration of the active material. The theory demonstrates that these differences are due to an intramolecular dissociation mechanism in the conjugated polymer systems and an intermolecular dissociation mechanism in the molecularly doped polymer systems.
\end{abstract}

Introduction. The opto-electronic properties of organic solids have gained a lot of attention over the last few years due to their potential application in, e.g., light-emitting diodes and solar cells (for recent reviews, see [1]). The organic solids to be discussed in this paper are molecularly doped polymers (MDPs) and conjugated polymers (CPs). In MDPs, optically active dye molecules are dispersed in an inert polymer matrix. In CPs the optical activity stems from the main chain of the polymer itself. Experiments indicate similarities between CPs and MDPs, which implies that, due to disorder, different segments of the CP chain act as independent molecules [2].

The electric field induced photoluminescence (PL) quenching experiments by Deussen et al. on CP blends [3] and on MDPs [4] allow for a direct comparison of these materials. The systems under study consist of a polycarbonate (PC) matrix, blended either with the CP poly(phenyl-p-phenylene vinylene) (PPPV) or with the dye molecule tris(stilbene)amine (TSA), which has a chemical structure related to that of PPPV (see insets to Fig. 1). In both systems, the PL quenching has been ascribed to the field induced dissociation of a molecular exciton into separate charge carriers. However, clear differences have been observed, especially in the dependence of the PL quenching on the concentration $c$ of the active material. As shown in Fig. 1a, the PL quenching in the CP system has a finite value at very low $c$ and saturates at $c>30 \%$. In the MDP system, the PL quenching is zero at $c \simeq 0$ and does not saturate with $c$ (see Fig. 1b). The purpose of the present paper is to give an outline of the theory of exciton migration and dissociation and to discuss how the observed differences in the PL quenching can be related to distinct exciton dissociation mechanisms.

\footnotetext{
$\left.{ }^{1}\right)$ phone: +31-40-2744411; Fax: +31-40-2743365; e-mail: vissenbe@natlab.research.philips.com
} 

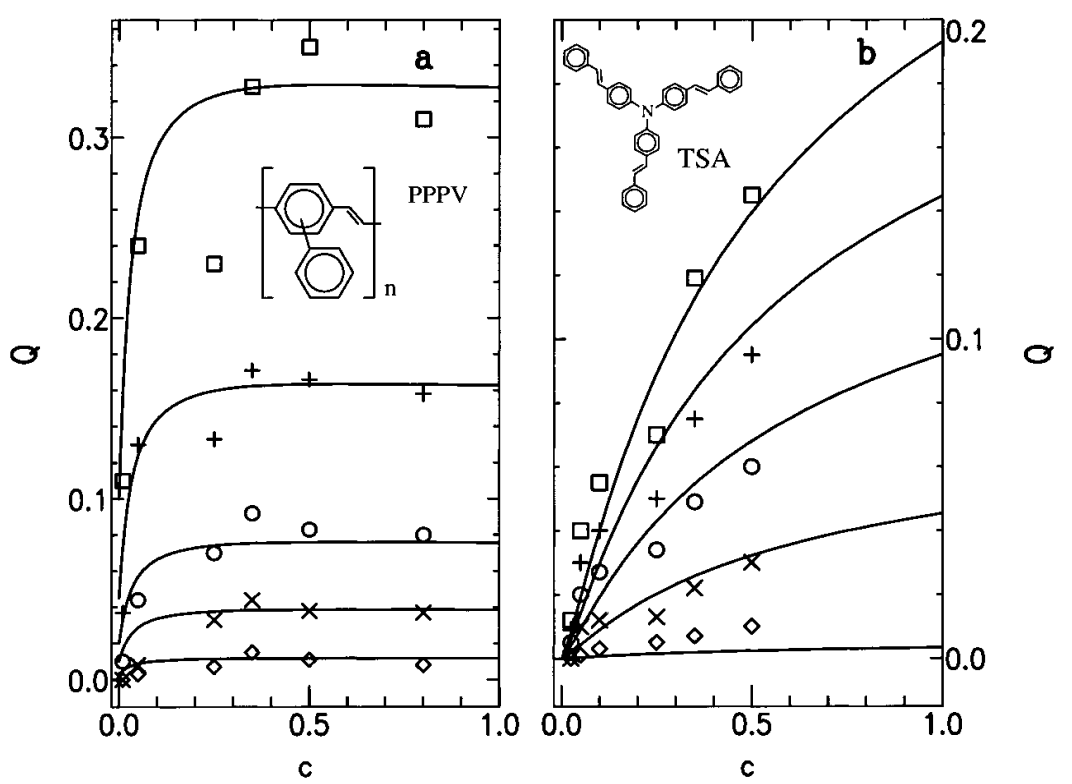

Fig. 1. Photoluminescence quenching $Q$ as a function of concentration $c$ of a) PPPV and b) TSA for an electric field $E=0.5,1.0,1.5,2.0$, and $2.5 \mathrm{MV} / \mathrm{cm}$ (from bottom to top). The experimental data, indicated by symbols, are taken from $[3,4]$. The curves follow from theory. The insets to the figures give the chemical structure of PPPV and TSA, respectively

Exciton Dynamics. Let us briefly describe the theory of exciton dynamics in a disordered molecular solid $[5,6]$. We consider a system consisting of localized states $i$, with random positions $\mathbf{R}_{i}$ and exciton energies $\varepsilon_{i}$, distributed according to the density of states $\varrho(\varepsilon)$. The occupational probability $f_{i}(t)$ of the site $i$ at time $t$ is described by the master equation

$$
\frac{\partial}{\partial t} f_{i}(t)=-\sum_{j \neq i} W_{j i} f_{i}(t)+\sum_{j \neq i} W_{i j} f_{j}(t)-\lambda_{i} f_{i}(t),
$$

with $\lambda_{i}$ the exciton decay rate on site $i$ and $W_{i j}=v_{0}\left(R_{0} /\left|\mathbf{R}_{i}-\mathbf{R}_{j}\right|\right)^{6} \theta\left(\varepsilon_{j}-\varepsilon_{i}\right)$ the Förster transition rate from site $j$ to $i$ [7]. Here, $v_{0}$ is the nearest-neighbor jump frequency, $R_{0}$ is the nearest-neighbor distance, and $\theta(x)=1$ if $x>0, \theta(x)=0$ otherwise. As the disorder in the site energies is usually much larger than the thermal energy, only jumps downward in energy are considered. The radiative recombination of excitons is described by a site-independent rate $\lambda$. In the presence of an electric field $E$, excitons may dissociate into separate charge carriers. This effect is included in our theory by increasing the exciton decay rate on a fraction $\alpha$ of the sites to $\lambda+\lambda_{\mathrm{d}}$, where $\lambda_{\mathrm{d}}$ denotes the exciton dissociation rate. The exciton dissociation mechanism determines how $\alpha$ and $\lambda_{\mathrm{d}}$ depend on $E, \varepsilon$, the exciton binding energy $E_{\mathrm{b}}$, and the local configuration of the molecules.

The stationary $\mathrm{PL}$ intensity under constant illumination (absorption rate $\beta$ ) can be written as

$$
L=\lambda \beta \int \mathrm{d} t \mathrm{~d} \varepsilon \varrho(\varepsilon)\left[G_{1}(\varepsilon, t)+\int \mathrm{d} \mathbf{R} \mathrm{d} \varepsilon^{\prime} \varrho\left(\varepsilon^{\prime}\right) G_{2}\left(\varepsilon, \varepsilon^{\prime}, \mathbf{R}, t\right)\right] .
$$


The local Green function $G_{1}(\varepsilon, t)$ denotes the average probability that an exciton remains at its initial site, and is given by [7]

$$
G_{1}(\varepsilon, t)=\left[(1-\alpha)+\alpha \mathrm{e}^{-\lambda_{\mathrm{d}} t}\right] \mathrm{e}^{-\lambda t-n(\varepsilon) 4 / 3 \pi R_{0}^{3} \sqrt{\pi \nu_{0} t}},
$$

with $n(\varepsilon)=\int_{-\infty}^{\varepsilon} \mathrm{d} \varepsilon^{\prime} \varrho\left(\varepsilon^{\prime}\right)$ the density of sites with energies below $\varepsilon$. In Eq. (2), the nonlocal Green function $G_{2}\left(\varepsilon, \varepsilon^{\prime}, \mathbf{R}, t\right)$ denotes the average probability that an exciton has migrated towards a site $\varepsilon$ at a distance $\mathbf{R}$ from the initial site $\varepsilon^{\prime}$. This function can be decomposed into local Green functions [5]. The stationary PL quenching

$$
Q=[L(E=0)-L(E \neq 0)] / L(E=0)
$$

can be calculated from Eqs. (2) and (3).

Results. In [5], we have modeled the PL quenching in the PPPV/PC blends, using $\alpha$ and $\lambda_{\mathrm{d}}$ as fit parameters. The result is shown in Fig. 1a. It is found that the saturation of $Q$ at high $c$ can only be obtained with $\alpha$ and $\lambda_{\mathrm{d}}$ independent of $c$. The concentration dependence of $Q$ can then be understood as follows. At low $c$, the excitons stay on their initial site and $Q=\alpha \lambda_{\mathrm{d}} /\left(\lambda+\lambda_{\mathrm{d}}\right)$. When $c$ increases, excitons start to migrate and the probability of an encounter with a dissociation site increases. At high $c$, the migration of excitons becomes sufficiently fast for excitons to escape from a dissociation site before dissociation takes place, so that $Q$ saturates.

To our opinion, the concentration independence of $\alpha$ and $\lambda_{\mathrm{d}}$ demonstrates that the exciton dissociation is an intramolecular process. This implies that the electron and hole are separated along the CP chain. This opinion has been commented upon by Conwell [8], who attributes the saturation of $Q$ with $c$ to the occurrence of phase segregation in the polymer blend. However, we think that the good agreement with stationary as well as with transient experiments remains a strong indication of the validity of our model [9].

We have analyzed the PL quenching data on the TSA/PC system within the same theoretical framework [10]. However, in this material the dissociation must be of intermolecular nature: one of the charge carriers tunnels towards a neighboring TSA molecule. The probability of finding a suitable nearest neighbor depends strongly on the concentration $c$ of TSA molecules, as the tunneling rate decays exponentially with the inter site distance. Consequently, the dissociation probability $\alpha$ (and hence the PL quenching $Q$ ) vanishes at low $c$ and increases monotonically with $c$ (see Fig. 1b).

In conclusion, we have presented a theory to describe electric field induced PL quenching experiments, which demonstrates how the dependence of $Q$ on $c$ reflects the migration and dissociation of excitons in disordered molecular solids.

Acknowledgements. We acknowledge interesting discussions with G. H. L. Brocks and useful correspondence with H. Bässler. This work has been supported by the Dutch Science Foundation NWO/FOM.

\section{References}

[1] A. J. Epstein and Y. YAng (Eds.), Mater. Res. Bull. 22, 6 (1997).

[2] H. Bässler, V. Brandl, M. Deussen, E. O. GöBel, R. Kersting, H. Kurz, U. Lemmer, R. F. Mahrt, and A. Ochse, Pure and Appl. Chem. 67, 377 (1995). 
[3] M. Deussen, M. Scheidler, and H. Bässler, Synth. Met. 73, 123 (1995).

[4] M. Deussen, P. Haring Bolivar, G. Wegmann, H. Kurz, and H. Bässler, Chem. Phys. 207, 147 (1996).

[5] M. C. J. M. Vissenberg and M. J. M. De Jong, Phys. Rev. Lett. 77, 4820 (1996).

[6] B. Movaghar, B. Ries, and M. Grünewald, Phys. Rev. B 34, 5574 (1986).

M. Grünewald and B. Movaghar, J. Phys.: Condensed Matter 1, 2521 (1989).

[7] Th. Förster, Z. Naturf. 4a, 321 (1949).

[8] E. M. Conwell, Phys. Rev. Lett. 78, 4301 (1997).

[9] M. C. J. M. Vissenberg and M. J. M. De Jong, Phys. Rev. Lett. 78, 4302 (1997).

[10] M. C. J. M. Vissenberg and M. J. M. De Jong, Phys. Rev. B, to be published. 